\title{
Pathogenic effector functions of ACPA: Where do we stand?
}

\author{
René Toes, ${ }^{\oplus 1}$ David S Pisetsky ${ }^{2}$
}

\section{INTRODUCTION}

In the late 1990 s, the discovery that citrullinated proteins are targeted by autoantibodies known as anti-citrullinated protein antibodies (ACPA) revolutionised the study of rheumatoid arthritis (RA). Because of the high specificity of ACPA for RA, investigators began experiments to determine whether ACPA have a direct pathogenetic role in arthritis or whether these antibodies more simply reflect underlying $\mathrm{T}$-cell and $\mathrm{B}$-cell responses without themselves impacting on joint inflammation and damage. The debate on the role of ACPA has been extensive and has now entered a new phase with the publication of correction notes appearing in this issue $\mathrm{e}^{12}$ of Annals of the Rheumatic $(A R D)$. These notes concern papers that appeared in the journal in $2016^{3} 4$ and follow a retraction note and correspondence in other journals. ${ }^{5-7}$

In these notes, the study authors indicate that the monoclonal antibodies used in their experiments on ACPA pathogenicity did not, in fact, react with citrullinated proteins and/or peptides. As such, the putative functional activities of these monoclonals must have resulted from mechanisms other than antibody binding to a citrullinated target. The implications of these corrections and retractions for models of pathogenesis are wide ranging and call into question the nature of ACPA involvement in manifestations of RA. Because of the importance of these issues for the scientific community, in this editorial, we will review the role of ACPA in $\mathrm{RA}$ and the salient issues in establishing the pathogenicity of these autoantibodies.

\section{ACPA AS BIOMARKERS IN RA}

ACPA are unique biomarkers for RA, a serious form of arthritis characterised by inflammation, pain and, unless treated effectively, damage of cartilage and bone.

\footnotetext{
'Department of Rheumatology, Leiden University Medical Center, Leiden, The Netherlands

${ }^{2}$ Department of Medicine, Duke University Medical Center and Medical Research Service, VA Medical Center, Durham, North Carolina, USA
}

Correspondence to Dr René Toes, Rheumatology, Leiden University Medical Center, Leiden 2333 ZA, Netherlands; r.e.m.toes@lumc.nl
As shown in elegant biochemical studies, ACPA target proteins that contain the amino acid citrulline, a post-translational modification (PTM) of the amino acid arginine mediated by peptidyl arginine deiminase enzymes ${ }^{89}$; these antibodies are also called anti-cyclic citrullinated peptide (CCP) antibodies because of their binding to CCP, a synthetic peptide used as a test antigen in immunoassays. ${ }^{89}$ ACPA provide an important criterion in diagnosis and classification. $^{10}$

While the strong association of ACPA with RA suggests a role in joint inflammation, proof of pathogenicity can be challenging and requires both direct and indirect evidence. Analogous to Koch's postulates for infectious disease, Witebsky's postulates for autoimmune disease systemise the experimental evidence needed to demonstrate that a clinical condition is autoimmune and that an autoimmune response (autoantibody or B/T cell) causes pathology. ${ }^{11}$ For ACPA, the challenge is especially great since ACPA are highly cross-reactive and bind a wide variety of citrullinated proteins; these proteins include citrullinated versions of enolase, fibrinogen, vimentin, collagen and histones, among others. ${ }^{12} 13$ The relevant target antigen in vivo and its tissue localisation are unknown, although joint tissue in RA shows evidence of citrullination. $^{14}$

For an autoantibody-mediated disease, pathogenicity is based on the presence of an autoantibody in affected patients; the transfer of disease to an animal by a source of antibodies, including monoclonal antibodies derived from blood or tissue; and induction of disease in an animal model by immunisation with the putative autoantigen. In some situations, an in vitro system can provide a model for a cellular response key to pathogenesis if the relationship to clinical findings is clear. The use of animal models is frequently informative, although it is possible that an autoantibody requires a particular setting (eg, low level of tissue injury or inflammation, the presence of other autoantibodies) for the pathogenicity to be manifest; in this case, the use of wild-type, unmanipulated mice may miss a pathogenic effect. ${ }^{15}$
POSTULATED MECHANISMS FOR ACPA PATHOGENICITY

For ACPA, two main mechanisms for disease induction or exacerbation have been proposed. ${ }^{16-18}$ The first mechanism involves the interaction of ACPA and a citrullinated protein to form immune complexes (IC). IC can activate the complement system and induce the release of chemotactic factors such as $\mathrm{C} 3 \mathrm{a}$ and C5a to allow recruitment of immune cells to a local site. These cells can subsequently be activated in a fragment crystallisable (Fc) $\gamma$-receptor-dependent manner. This activation can lead to the production of cytokines and other pro-inflammatory mediators, culminating in inflammation in general, although arthritis could occur preferentially if the citrullinated proteins are located in the joint or if ACPA production occurs primarily in that local environment. The ability of an ACPA to form an IC may depend on the specificity of the antibody as well as the nature of the citrullinated protein that can be bound in complexed form. IC, including ACPA containing IC, can also bind rheumatoid factor (RF), an autoantibody against IgG which very frequently co-occurs with ACPA; like that of ACPA, the expression of RF can precede the clinical manifestations of the disease and is also important for diagnostic and classification purposes. $^{1019-21}$

A more novel mechanism proposed for ACPA pathogenicity relates to the ability of autoantibodies to serve as agonists for a receptor-mediated response. Indeed, these studies, which are the subject of the correction notes, presented intriguing observations indicating that ACPA can directly induce both pain and osteoclastogenesis; these are two cardinal features of RA that can impact on patient symptomatology as well as clinical outcomes. ${ }^{34}$ These studies involved transfer into mice of IgG purified from patient sera as well as monoclonal ACPA to assess pain responses and bone loss; studies on osteoclastogenesis involved in vitro culture systems as well. While both polyclonal and monoclonal antibody preparations produced similar findings in the $A R D$ papers, the authors have meanwhile revealed that the monoclonal antibodies used in these experiments do not bind citrullinated antigens. $^{5}$

In view of these issues, it is useful to review studies on the pathogenicity of ACPA and determine how studies with monoclonals without apparent ACPA activity could induce pathogenic events in either in vivo or in vitro studies. 


\section{THE EFFECTS OF ACPA ON}

INFLAMMATION, PAIN AND BONE LOSS

As noted, one potential mechanism by which ACPA can promote events in RA relates to their ability to form IC that can activate cells. Indeed, several studies have demonstrated that the Fc tail of ACPA can activate both Fc $\gamma$-receptor expressing cells and the complement system. ${ }^{16}{ }^{18}$ Since the expression of citrullinated antigens occurs in the tissue in vivo, including the synovial compartment, ${ }^{14}$ these studies suggest that ACPA-containing IC can induce inflammation in RA. Importantly, other studies have provided evidence that RF can enhance these effects in vitro, presumably by promoting IC formation via multivalent binding. ${ }^{19-21}$ These observations link the two characteristic serological findings in RA into one pathogenic pathway and are consistent with clinical findings that arthritis is more severe in patients who express both ACPA and RF. ${ }^{22}$

While ACPA could drive inflammation by complement system-mediated and Fc $\gamma$-receptor-mediated effects, other studies suggest a novel role of ACPA by direct binding to molecules expressed on the surface of cells; these molecules are presumably citrullinated. Indeed, the studies in ARD reported that ACPA can directly induce osteoclastogenesis, thereby contributing to bone loss and joint erosion that are typical for RA. Furthermore, studies by Wigerblad et al in ARD indicated that ACPA can induce pain in an in vivo mouse model by activating osteoclasts to release interleukin 8 and potentially other mediators involved in pain. ${ }^{34}$ The effects observed in these studies appeared to be specific for ACPA since human IgG preparations enriched for ACPA, induced pain behaviour in mice; in contrast, IgG preparations derived from ACPA-negative patients or $\operatorname{IgG}$ preparations depleted of ACPA were inactive.

Consistent with the principles embodied in Witebsky's postulates (which long preceded monoclonal antibody technology), studies with purified IgG from patient blood can provide at least circumstantial evidence for the pathogenicity of a particular antibody specificity. In the ARD publications, the use of monoclonal antibodies derived from patient sources provided perhaps more compelling evidence that the effects on pain and osteoclastogenesis result from direct ACPA binding. In these experiments, murinised monoclonal ACPA, but not monoclonal control antibodies, elicited pain-like behaviour that coincided with the ability to activate osteoclasts. Furthermore, since monomeric Fab fragments were reported to promote osteoclastogenesis, ${ }^{3} 23$ these effects appeared independent of the Fc region of the antibodies and, hence, from Fc $\gamma$-receptor triggering. This last notion is supported by the observation that only monoclonal ACPA displaying a certain epitope-specificity profile could activate osteoclasts, arguing that epitope specificity of the ACPA is crucially involved in the induction of osteoclastogenesis. ${ }^{3}$ As now known, however, these monoclonal antibodies do not have citrulline specificity. ${ }^{5}$

In view of the strong associations between ACPA and RA, the findings published in $A R D$ in 2016 attracted great interest as they could explain two prominent features of RA-joint pain and bone erosion in a disease-specific manner. As such, these observations entailed a paradigm shift in thinking about disease pathogenesis by suggesting that certain disease manifestations in RA could arise directly from RA-specific autoantibodies and do not entail IC formation. In this conceptualisation of RA, ACPA would resemble autoantibodies that directly activate or functionally perturb cells such as autoantibodies found in Graves' disease which bind the thyrotropin receptor stimulating the production of thyroxine and triiodothyronine.

In ascribing pathogenicity to an autoantibody in any disease, a relevant consideration is plausibility (ie, does clinical evidence support the proposed mechanism?). In RA, a number of clinical situations impact on the plausibility that ACPA are pathogenic either by IC formation or agonistic activity. The first concerns the apparent lack of transfer of disease during pregnancy despite the placental transfer of autoantibodies. The expression of ACPA in asymptomatic individuals during the phase of pre-disease autoimmunity is another clinical scenario to consider. Another circumstance relates to the continued expression of ACPA following successful treatment of RA that can diminish pain and halt bone loss. While some reduction in ACPA levels occurs with therapy, this reduction is limited. ${ }^{24}$ In these various settings, why does not the presence of ACPA lead to either pain or bone loss?

\section{RE-EVALUATION OF DATA ON ACPA PATHOGENICITY}

While the concept that ACPA are agonistic agents is intriguing, the new findings ask for a critical re-interpretation of this possibility. Notably, the authors who described the monoclonal ACPA with osteoclastogenic and pain-inducing potential notified the community that, in reassessing the original data, the antibodies in question showed 'no measurable affinity for the tested citrullinated peptides' as detailed in a recent correction ${ }^{7}$ and retraction note ${ }^{5}$; in other words, the monoclonal antibodies studied were not ACPA. This observation is in line with crystallographic studies of some of these monoclonal antibodies that could not confirm the binding of citrullinated peptides. ${ }^{25}$ While there is a major unknown - the identity of the antigens that led to a positive signal on synovial tissue and/or osteoclasts-these antibodies had, nevertheless, in vivo and in vitro effects. As also acknowledged by the authors in the two correction notes published in this issue of $A R D$, it is now clear that 'the functional results reported for these monoclonal antibodies cannot be attributed to reactivity against citrullinated proteins and/or peptides, but are due to other yet unknown mechanisms'. ${ }^{12}$

In studies on pathogenicity, determination of antibody specificity is crucial and requires appropriate assays to detect binding at relevant concentrations. For ACPA, several different antigens can be used for immunochemical determinations. These include citrullinated proteins and citrullinated peptides especially when presented in large arrays in which a multitude of sequences can be assessed. Other considerations in assessing antibody specificity relate to the detection method (eg, ELISA, surface plasmon resonance, western blotting) that may, in turn, be influenced by technical factors such as salt concentrations and even the nature of the buffer.

For monoclonal antibodies, polyreactivity and cross-reactivity (ie, ability to bind to multiple different antigens or the ability to bind to multiple citrullinated and/or carbamylated/acetylated antigens) may be a complicating factor. Polyreactivity may result from an aberrant 'pattern of antigen-driven selection' in the setting of autoimmunity. In autoimmune disease, the repertoires and signalling thresholds for both $\mathrm{T}$ and $\mathrm{B}$ cells may be abnormal, skewing the binding properties of induced antibodies. In addition to disturbances in B-cell and T-cell function, antigen drive by multiple different antigens may lead to patterns of reactivity unlike those of a conventional antibody response where antigen binding is more specific and has higher avidity. ${ }^{26-28}$ In this regard, since the nature of self-antigens in vivo is often not known, extrapolating from in vitro assays with purified antigens to that which occurs in vivo can be uncertain especially 


\section{A) Postulated mechanism for ACPA pathogenicity}

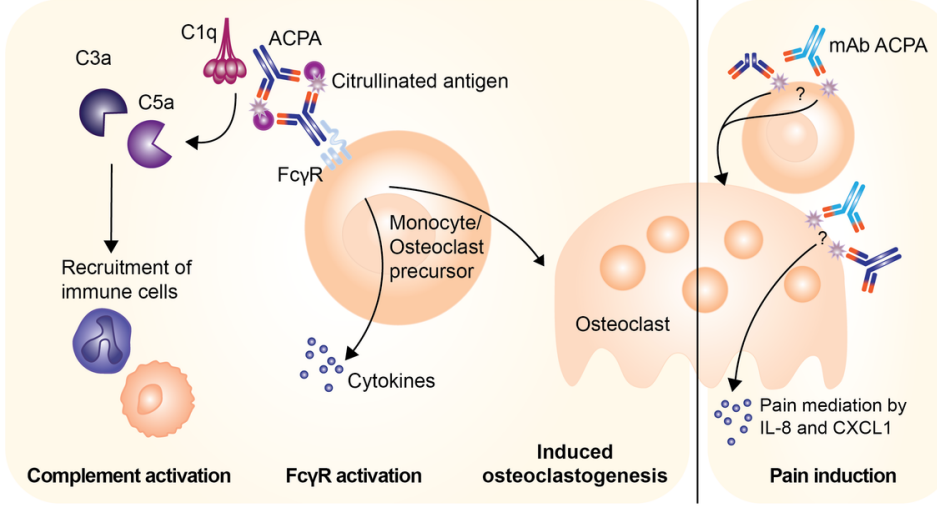

B) Issues in experimental design and performance
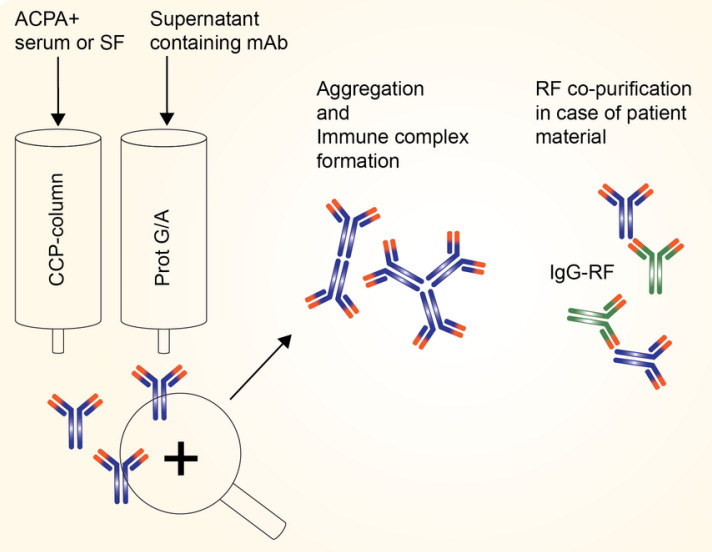

Endotoxins and non-endotoxin pyrogens

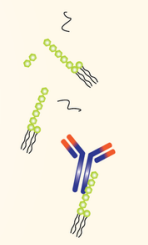

Figure 1 Operational and postulated principles in defining the pathogenic activity of polyclonal or monoclonal antibodies. (A) Postulated mechanism for ACPA pathogenicity either by polyclonal ACPA and/or monoclonal ACPA. Left side: confirmed autoantibody-mediated contributions to arthritis facilitated by complement system activation and Fc $\gamma$ receptor activation; ${ }^{16-18}$ right side: postulated contribution of ACPA to RA as now under debate indicated by correction notes published in this issue ${ }^{12}$ to papers published in $A R D^{34}$ as well as a preceding retraction and correspondence in other journals. ${ }^{5-7}$ The questions marks indicate the re-evaluation of the pathogenic activity of the monoclonal ACPA, as it is reassessed to be not specific for citrullination. (B) Potential issues occurring during antibody isolation of either ACPA from patient sera or supernatant containing recombinant monoclonal antibodies. Factors that can occur during purification: aggregation, immune complex formation, co-purification of RF in case of ACPA+ serum or synovial fluid and lastly introduction of endotoxin and non-endotoxin pyrogens. Light blue antibody: monoclonal ACPA; dark blue: ACPA from patient lgG fraction enriched for ACPA; asterisk: citrullinated protein; green antibody: RF IgG. ACPA, anti-citrullinated protein antibody; RA, rheumatoid arthritis; RF, rheumatoid factor.

when an antibody can bind to structurally similar molecules in a cross-reactive manner.

\section{ISSUES IN EXPERIMENTAL DESIGN AND PERFORMANCE}

Given these considerations as well as notices of correction and retraction, current data indicate that the biological effects observed with the monoclonal antibodies appear unrelated to the recognition of citrulline as a critical determinant of specificity. 5625 Hence, other mechanisms, unrelated to the recognition of citrullinated antigens, must have contributed to the results obtained (figure 1). Whether this situation also pertains to the effects of the affinity-purified polyclonal ACPA containing Ig preparations is more difficult to know. Importantly, however, the authors of the $2016 A R D$ papers on pain induction and osteoclastogenesis state that 'also these results have to be interpreted with caution waiting for additional mechanistic studies' ${ }^{12}$ With polyclonal antibodies, it is important to absorb reactivity with citrullinated versus control antigens to show that, in the first case, the biological effects are eliminated while, in the control condition, they are retained. Still, the binding of antibodies to affinity columns provides presumptive evidence of specificity, but does not prove it. Regardless, since the identification and molecular characterisation of any putative citrullinated receptor have not yet been accomplished, caution is needed in the interpretation of results with the polyclonal preparations as well as monoclonal antibodies. Of note, the avidity of ACPA appears to be relatively low, potentially limiting their ability to directly activate cellular receptors. ${ }^{29} 30$

While the relevant specificity of both the polyclonal and monoclonal antibody preparations remains an open question, the finding on the induction of pain and bone loss is nevertheless very interesting and would benefit from reasonable and plausible explanations to move the field forward. In retrospect, several possibilities could be at play. For example, it is possible that the monoclonal and polyclonal antibody preparations contained contaminants that by themselves might have caused biological effects. Myeloid cells, for example, are highly sensitive to endotoxin and non-endotoxin pyrogens; this sensitivity can differ from that of conventional endotoxin-detection kits, such as the Limulus Amebocyte Lysate test. Endotoxin and other pyrogens can be co-purified during protein isolation due to hydrophobic interactions, for example. ${ }^{31} 32$ Such events can occur variably among antibody preparations, explaining why some monoclonal antibodies or their respective purified Fab preparations activate osteoclasts, whereas others do not.

Another contributing factor relates to the immunochemical properties of an antibody molecule. Among the monoclonal antibodies that could induce osteoclastogenesis and joint pain, D10 and $\mathrm{B} 02$ were reported to show 'polyreactivity' towards at least two antigens out of three analysed (ie, to doublestranded DNA, insulin and/or lipopolysaccharide (LPS). This activity contrasts with that of at least one of the monoclonal antibodies which did not appear to elicit these effects (RA1276:01:C07). Likewise, because of aggregation that can occur during affinity purification or during the isolation of monoclonal antibodies, certain monoclonal antibody preparations can act as ICs to activate Fc $\gamma R$-positive cells; the production of heavy chain dimers can lead to the same effect. In this situation, batch-to-batch and IgG-to-IgG variations can occur. ${ }^{33-36}$ Such variability could also explain why 
some (monoclonal) antibody preparations induce effects in cellular systems, whereas others do not. Similarly, the presence of IgG-RF in IgG preparations purified from serum could also lead to confounding results.

It is important to consider that ACPA might be particularly prone to aggregation and interactions with pyrogens since they display some unique features including the presence of negatively charged, $\mathrm{N}$-linked glycans in the variable domain. ${ }^{3738}$ Also in this case, the composition of the $\mathrm{N}$-linked glycans present on recombinantly produced antibodies may vary substantially from batch-to-batch and/or between different antibodies when not controlled for and hence could impact on biological outcomes. ${ }^{39-41}$ The experience of one of us (RT) with isolated ACPA, monoclonal ACPA, as well as control antibody preparations is consistent with this possibility since we also obtained inconsistent and variable effects in similar studies addressing the osteoclastogenic effects of ACPA (unpublished data). In this context, variable effects seem to be present in the published experiments as well since, in a recent study, one of four monoclonal ACPA induced osteoclastogenesis slightly, two had no effect and the fourth inhibited osteoclastogenesis; as such, when antibodies are present together as may happen during disease, there may be no net effect, with one effect on osteoclast activation counteracted by another. ${ }^{42}$

Similarly, it has been reported that the osteoclastogenic potential of ACPA occurs only with cells from wild-type mice, but not with cells derived from Fc $\gamma$ - or Fc $\gamma$ RIII knockout mice, ${ }^{43}$ a notion that seems to conflict with the observation that Fab domains may be sufficient to mediate the effects described. In this respect, it might be relevant to also re-evaluate the reported osteoclastogenic potential of Fab fragment isolated against citrullinated vimentin. ${ }^{23}$ The observations obtained with cells from Fc $\gamma \mathrm{R}$ knockout mice are, however, in line with the findings that (heat)-aggregated IgGs display a strong osteoclastogenic potential; this potential may be more pronounced for aggregated IgG preparations enriched for ACPA, perhaps related to the reduced sialylation of the $\mathrm{N}$-linked $\mathrm{Fc}$ glycans which enhances Fc $\gamma R$ binding. ${ }^{44}$

\section{IMPLICATIONS FOR THE FUTURE}

The publication of retraction and correction notes is always unfortunate because

Box 1 Considerations for defining the pathogenic activity of either polyclonal or monoclonal antibodies

- Assess antibody activity using well-validated assays especially those that are clinically useful.

- Validate assays sufficiently to ensure that they detect specific antibody binding and not non-specific interactions.

- Assure antibody specificity by direct binding and inhibition studies of polyclonal and monoclonal antibodies.

- For affinity purification, consider use of different antigens (eg, different citrullinated proteins) for the affinity matrix and assess both bound and unbound fractions for antibody binding and functional activity.

- Remove aggregates by ultracentrifugation or size-exclusion chromatography.

- Assess for contamination by endotoxin and related material.

- For in vitro studies, perform dose-response curves and assess activity at relevant antibody concentrations.

- For in vivo studies, assess activity in different strains of mice including knockout mice to define mechanisms.

- For in vivo studies, consider the use of models in which subclinical arthritis is induced.

- For both in vivo and in vitro studies, consider the effects of rheumatoid factor.

- In case of agonistic antibodies binding to cellular receptors, clone the receptor from the target cells for expression in reporter cells to mimic agonistic activity.

of the effects on investigators especially when unforeseen technical issues have affected experiments. Beyond effects on investigators, however, retractions and corrections, especially if delayed, also negatively impact the scientific community as a whole since investigators who were attracted by reported findings may have invested time and effort to explore these observations, leading them in a perhaps wrong direction or deterring them from pursuing other potential mechanisms. Correcting the literature will also take time especially as novel ideas often receive prominence in reviews and other articles.

While disappointing and regrettable, these circumstances nevertheless provide an opportunity to rethink concepts as well as improve experimental designs. Given publication of several studies on agonist activities of ACPA, these considerations do not exclude the possibility that ACPA (or even a related specificity such as anti-malondialdehyde [monoclonal] antibodies as recently proposed) ${ }^{45}$ can directly activate osteoclasts to induce bone resorption and pain; providing new evidence in support of this phenomenon is crucial, especially to explain the activity of polyclonal antibody preparations.

Adding to the complexity of the ACPA system is the extensive cross-reactivity of these autoantibodies towards different citrullinated antigens as well as other PTMs expressed by proteins. ${ }^{42} 4647$ As these different modifications are recognised with varying avidities, attributing biological effects of monoclonal or polyclonal antibodies to the recognition of citrulline alone is difficult and it can be debated whether citrulline specificity and/or the 'Peptidyl Arginine Deiminase-Citrulline' pathway should be considered the predominant or indeed only auto-reactive phenomenon. In this respect, it is important to note that recent epidemiological data may not support the postulated connection between smoking and formation of citrullinated proteins and a breach of tolerance to citrullinated antigens ${ }^{48}$; thus, smoking may be associated with the concurrent presence of multiple autoantibodies in RA rather than with ACPA per se. ${ }^{49-51}$ Ultimately, it will be essential to delineate fully the autoantibody repertoire in RA and to clone, express and to define at the molecular level the possible cellular receptors targeted by ACPA, assessing the relevant PTM. In view of the experience with ACPA, we would suggest the following operational principles in defining the pathogenic activity of either polyclonal or monoclonal antibodies (Box 1).

\section{CONCLUSIONS}

The recent information on the binding properties of monoclonal antibodies once considered to be ACPA will require a period of reflection and re-evaluation of prior results on the potential pathogenicity of ACPA in RA. Nevertheless, the data presented in a number of papers suggest that autoantibodies can mediate events 
in RA by diverse mechanisms, including IC formation and, possibly, direct agonist activity. Clearly, as the field moves forward, the claims that ACPA can activate cells implicated in pathogenesis should receive more extensive and rigorous experimental support before they can be accepted. Nonetheless, as our understanding of the unique features of ACPA and other autoantibodies grows, these mechanisms will undoubtedly undergo further investigation. In the quest to understand the possible contribution of ACPA to disease pathogenesis, these efforts will provide new and stimulating insights into the pathways contributing to RA pathogenesis and hopefully allow new approaches to diagnosis, staging and treatment.

\section{Handling editor Josef S Smolen}

Acknowledgements We thank Dr Hans Ulrich Scherer for critically reading the manuscript and Ms Lise Hafkenscheid for drawing of figure 1.

Funding The authors have not declared a specific grant for this research from any funding agency in the public, commercial or not-for-profit sectors.

Competing interests None declared.

Patient consent for publication Not required.

Provenance and peer review Commissioned; internally peer reviewed.

(C) Author(s) (or their employer(s)) 2019. No commercial re-use. See rights and permissions. Published by BMJ.

\section{(D) Check for updates}

To cite Toes R, Pisetsky DS. Ann Rheum Dis 2019;78:716-721.

Ann Rheum Dis 2019;78:716-721.

doi:10.1136/annrheumdis-2019-215337

\section{REFERENCES}

1 Correction to the paper by Wigerblad. Ann Rheum Dis 2016;75:730-8.

2 Correction to the paper by Krishnamurty. Ann Rheum Dis 2016;75:721-9.

3 Krishnamurthy A, Joshua V, Haj Hensvold A, et al. Identification of a novel chemokine-dependent molecular mechanism underlying rheumatoid arthritisassociated autoantibody-mediated bone loss. Ann Rheum Dis 2016:75:721-9.

4 Wigerblad G, Bas DB, Fernades-Cerqueira C, et al. Autoantibodies to citrullinated proteins may induce joint pain independent of inflammation. Ann Rheum Dis 2016;75:730-8.

5 Amara K, Steen J, Murray F, et al. Retraction: monoclonal IgG antibodies generated from jointderived $B$ cells of RA patients have a strong bias toward citrullinated autoantigen recognition. J Exp Med 2019:216.

6 Holmdahl R. The specificity of monoclonal AntiCitrullinated protein antibodies: comment on the article by Steen et al. Arthritis Rheumatol 2019;71:324-5.

7 Malmström V, Steen J, Klareskog L. Reply. Arthritis Rheumatol 2019:71:325-7.

8 Girbal-Neuhauser E, Durieux JJ, Arnaud M, et al. The epitopes targeted by the rheumatoid arthritisassociated antifilaggrin autoantibodies are posttranslationally generated on various sites of (pro)filaggrin by deimination of arginine residues. J Immunol 1999;162:585-94.

9 Schellekens GA, de Jong BA, van den Hoogen $\mathrm{FH}$, et al. Citrulline is an essential constituent of antigenic determinants recognized by rheumatoid arthritis-specific autoantibodies. J Clin Invest 1998; 101:273-81.

10 Aletaha D, Neogi T, Silman AJ, et al. 2010 rheumatoid arthritis classification criteria: an American College of Rheumatology/European League against rheumatism collaborative initiative. Ann Rheum Dis 2010;69:1580-8.

11 Rose NR, Bona C. Defining criteria for autoimmune diseases (Witebsky's postulates revisited). Immunol Today 1993:14:426-30.

12 Chandra PE, Sokolove J, Hipp BG, et al. Novel multiplex technology for diagnostic characterization of rheumatoid arthritis. Arthritis Res Ther 2011;13.

13 Ioan-Facsinay A, el-Bannoudi H, Scherer HU, et al. Anticyclic citrullinated peptide antibodies are a collection of anti-citrullinated protein antibodies and contain overlapping and non-overlapping reactivities. Ann Rheum Dis 2011;70:188-93.

14 Vossenaar ER, Smeets TJM, Kraan MC, et al. The presence of citrullinated proteins is not specific for rheumatoid synovial tissue. Arthritis Rheum 2004:50:3485-94

15 Kuhn KA, Kulik L, Tomooka B, et al. Antibodies against citrullinated proteins enhance tissue injury in experimental autoimmune arthritis. $J$ Clin Invest 2006;116:961-73.

16 Clavel C, Nogueira L, Laurent L, et al. Induction of macrophage secretion of tumor necrosis factor alpha through Fcgamma receptor Ila engagement by rheumatoid arthritis-specific autoantibodies to citrullinated proteins complexed with fibrinogen. Arthritis Rheum 2008:58:678-88

$17 \mathrm{Ji} \mathrm{H}$, Ohmura K, Mahmood U, et al. Arthritis critically dependent on innate immune system players. Immunity 2002;16:157-68.

18 Trouw LA, Haisma EM, Levarht EWN, et al. Anti-cyclic citrullinated peptide antibodies from rheumatoid arthritis patients activate complement via both the classical and alternative pathways. Arthritis Rheum 2009;60:1923-31.

19 Anquetil F, Clavel C, Offer G, et al. IgM and IgA rheumatoid factors purified from rheumatoid arthritis sera boost the Fc receptor- and complementdependent effector functions of the disease-specific anti-citrullinated protein autoantibodies. J Immunol 2015; 194:3664-74

20 Laurent L, Anquetil F, Clavel C, et al. IgM rheumatoid factor amplifies the inflammatory response of macrophages induced by the rheumatoid arthritisspecific immune complexes containing anticitrullinated protein antibodies. Ann Rheum Dis 2015;74:1425-31.

21 Sokolove J, Johnson DS, Lahey L, et al. Rheumatoid factor as a potentiator of anti-citrullinated protein antibody-mediated inflammation in rheumatoid arthritis. Arthritis Rheumatol 2014;66:813-21.

22 Aletaha D, Alasti F, Smolen JS. Rheumatoid factor, not antibodies against citrullinated proteins, is associated with baseline disease activity in rheumatoid arthritis clinical trials. Arthritis Res Ther 2015;17.

23 Harre U, Georgess D, Bang $\mathrm{H}$, et al. Induction of osteoclastogenesis and bone loss by human autoantibodies against citrullinated vimentin. J Clin Invest 2012;122:1791-802

24 Böhler C, Radner H, Smolen IS, et al. Serological changes in the course of traditional and biological disease modifying therapy of rheumatoid arthritis. Ann Rheum Dis 2013:72:241-4

$25 \mathrm{Ge} \mathrm{C}, \mathrm{Xu}$ B, Liang B, et al. Structural basis of crossreactivity of Anti-Citrullinated protein antibodies. Arthritis Rheumatol 2019;71:210-21.

26 Aitcheson CT, Peebles C, Joslin F, et al. Characteristics of antinuclear antibodies in rheumatoid arthritis. Reactivity of rheumatoid factor with a histone- dependent nuclear antigen. Arthritis Rheum 1980:23:528-38

27 Pisetsky DS, Hoch SO, Klatt CL, et al. Specificity and idiotypic analysis of a monoclonal anti-Sm antibody with anti-DNA activity. J Immunol 1985;135:4080-5.

28 Rubin RLet al. Multiple autoantigen binding capabilities of mouse monoclonal antibodies selected for rheumatoid factor activity. J Exp Med 1984;159:1429-40

29 Suwannalai $\mathrm{P}$, Scherer HU, van der Woude D, et al. Anti-citrullinated protein antibodies have a low avidity compared with antibodies against recall antigens. Ann Rheum Dis 2011:70:373-9.

30 Usinger WR, Lucas AH. Avidity as a determinant of the protective efficacy of human antibodies to pneumococcal capsular polysaccharides. Infect Immun 1999:67:2366-70.

31 Dullah EC, Ongkudon CM. Current trends in endotoxin detection and analysis of endotoxin-protein interactions. Crit Rev Biotechnol 2017;37:251-61.

32 Warger T, Hilf N, Rechtsteiner G, et al. Interaction of TLR2 and TLR4 ligands with the N-terminal domain of Gp96 amplifies innate and adaptive immune responses. J Biol Chem 2006;281:22545-53.

33 Bondos SE, Bicknell A. Detection and prevention of protein aggregation before, during, and after purification. Anal Biochem 2003;316:223-31.

34 van der Kant R, Karow-Zwick AR, Van Durme J et al. Prediction and reduction of the aggregation of monoclonal antibodies. J Mol Biol 2017;429:1244-61.

35 Li W, Prabakaran P, Chen W, et al. Antibody aggregation: insights from sequence and structure Antibodies 2016;5.

36 Chung HH, Buck L, Daris K, et al. Investigation of the free heavy chain homodimers of a monoclonal antibody. Biotechnol Prog 2018;34:738-45.

37 Hafkenscheid L, Bondt A, Scherer HU, et al. Structura analysis of variable domain glycosylation of AntiCitrullinated protein antibodies in rheumatoid arthritis reveals the presence of highly sialylated glycans. Mol Cell Proteomics 2017:16:278-87.

38 Rombouts Y, Willemze A, van Beers JJBC, et al Extensive glycosylation of ACPA-IgG variable domains modulates binding to citrullinated antigens in rheumatoid arthritis. Ann Rheum Dis 2016;75:578-85.

39 Pacis E, Yu M, Autsen J, et al. Effects of cell culture conditions on antibody N-linked glycosylation--what affects high mannose 5 glycoform. Biotechnol Bioeng 2011;108:2348-58

40 Planinc A, Dejaegher B, Vander Heyden Y, et al. Batch-to-batch N-glycosylation study of infliximab, trastuzumab and bevacizumab, and stability study of bevacizumab. Eur J Hosp Pharm 2017;24:286-92.

41 van Berkel PHC, Gerritsen J, Perdok G, et al. N-linked glycosylation is an important parameter for optimal selection of cell lines producing biopharmaceutical human IgG. Biotechnol Prog 2009:25:244-51.

42 Steen J, Forsström B, Sahlström P, et al. Recognition of amino acid motifs, rather than specific proteins, by human plasma cell-derived monoclonal antibodies to posttranslationally modified proteins in rheumatoid arthritis. Arthritis Rheumatol 2019;71:196-209.

43 Krishnamurthy A, Steen J, Gronwall C, et al. RAAssociated antibodies targeting post translational modification have different Osteoclastogenetic potential. Arthritis \& Rheumatology 2017;69.

44 Harre U, Lang SC, Pfeifle R, et al. Glycosylation of immunoglobulin $\mathrm{G}$ determines osteoclast differentiation and bone loss. Nat Commun 2015;6.

45 Grönwall C. Amara K, Hardt U, et al. Autoreactivity to malondialdehyde-modifications in rheumatoid arthritis is linked to disease activity and synovial pathogenesis. J Autoimmun 2017;84:29-45.

46 Lloyd KA, Wigerblad G, Sahlström P, et al. Differential AcpA binding to nuclear antigens reveals a PAD-Independent pathway and a distinct subset of acetylation cross-reactive autoantibodies in rheumatoid arthritis. Front Immunol 2018;9. 
47 Shi J, Willemze A, Janssen GMC, et al. Recognition of citrullinated and carbamylated proteins by human antibodies: specificity, cross-reactivity and the 'AMCSenshu' method. Ann Rheum Dis 2013;72:148-50.

48 Malmström V, Catrina Al, Klareskog L. The immunopathogenesis of seropositive rheumatoid arthritis: from triggering to targeting. Nat Rev Immunol 2017; 17:60-75.
49 Hedström AK, Rönnelid J, Klareskog L, et al. Smoking, HLA-genes and serology in rheumatoid arthritis; complex relationships investigated in the Swedish EIRA case-control study. Arthritis Rheumatol 2019. doi:10.1002/art.40852. [Epub ahead of print: 11 Feb 2019].

50 Murphy D, Mattey D, Hutchinson D. Anti-citrullinated protein antibody positive rheumatoid arthritis is primarily determined by rheumatoid factor titre and the shared epitope rather than smoking per se. PLOS One 2017;12:e0180655.

51 van Wesemael TJ, Ajeganova S, Humphreys J, et al. Smoking is associated with the concurrent presence of multiple autoantibodies in rheumatoid arthritis rather than with anti-citrullinated protein antibodies per se: a multicenter cohort study. Arthritis Res Ther 2016;18. 